\title{
El promontorio y el puerto, el avistamiento y la acción ofensiva. Propuestas tipológicas de torres marítimas para un mismo problema geográfico: los puertos de Jávea y Moraira en el litoral valenciano (1562-1596)
}

\section{Antoni Banyuls Pérez}

Universidad de Alicante, Alicante, España, antonibanyuls@gmail.com

\begin{abstract}
The various geographical conditions of coastal relief demanded adopt, in turn, different solutions for fortification. One of these geographical problems, faced quite often, was the existence of a promontory and a port to his coat, like royal engineer Cristóbal Antonelli pointed in a drawing of 1596 on the Cape and the port of Moraira on the valencian coast.On the one hand it was necessary to supervise the coast and moreover, repel the enemy access to ports ships, all through maritime towers strategically located in coastal promontories. The analysis of this particular problem in coastal fortification in different areas allows the comparison of the different typologies of towers and proposed solutions to existing problems.
\end{abstract}

Keywords: Promontories, ports, maritime towers, typologies.

\section{Introducción}

En 1596 el ingeniero real Cristóbal Antonelli en un dibujo que representaba -visto desde el mar- el promontorio y la ensenada que forman el cabo y el puerto de Moraira en el litoral valenciano, vino a señalar -en aislarlos de la continuidad del frente marítimo- la existencia de un especifico problema de condición geográfica en la fortificación del litoral que exigía dar solución a exigencias de muy distinta índole: por una parte garantizar la continuidad del sistema de vigilancia en la altura del promontorio $\mathrm{y}$, por otra, la de repeler con artillería las naves enemigas en su tentativa de acceso al abrigo del puerto. Su propuesta, plasmada en la referida pintura, pasaba por proponer la existencia de dos torres de diferente tipología, en correspondencia con su emplazamiento y la función asignada que tendrían que desempeñar. Las torres atalayas edificadas en la costa valenciana entre $1553 \mathrm{y}$ 1558 a instancias del virrey Bernardino de Cárdenas, duque de Maqueda, tenían como fin el avistamiento de la presencia de naves enemigas $\mathrm{y}$, en la medida de lo posible, la vigilancia y descubrimiento de aquellos fondeaderos y calas que les pudieran servir como refugio. Su continuidad en el borde litoral e integración en un único sistema permitía la rápida comunicación de los avisos para poner en alerta a la población y en armas a las compañías de milicia (Boira, 2007). Con este objetivo el emplazamiento de estas torres, por lo general, atendió a la implantación más favorable en los lugares más elevados del relieve costero con el fin de poder obtener la mayor efectividad posible con el mínimo número de estas torres.

Otras torres como las que tenían por finalidad defender los puertos y aquellos otros fondeaderos que servían habitualmente como lugar de desembarco por sus favorables condiciones naturales, debían desempeñar una acción ofensiva mediante el uso de artillería. Este cometido de la torre como fuerza de repulsa 
exigía situarse, por el contrario, en una cota topográfica a más baja altura que permitiese tener a su alcance la amplitud de la ensenada. En función de este espacio a proteger estaría el número y/o calibre de las piezas de artillería necesarias que determinarían, a su vez, los recursos humanos y su arquitectura.

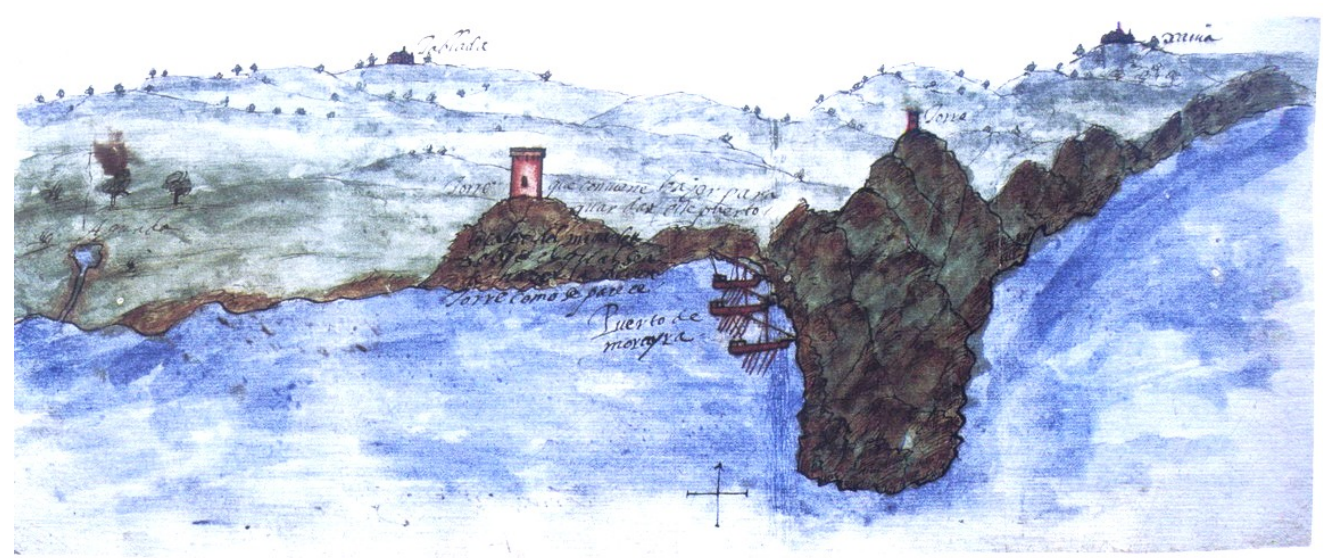

Fig. 1- Cristóbal Antonelli. Pintura del puerto de Moraira, ca. 1596 (España. Ministerio de Educación, Cultura y Deporte. Archivo de la Corona de Aragón, MP-23/3)

Es relativamente frecuente que en el relieve costero tenga lugar esta doble condición geográfica de promontorio y fondeadero a su abrigo que reflejaba el dibujo de Cristóbal Antonelli y, por ello, que surgiese la necesidad de tener que afrontar su fortificación muchas veces con más de una torre. El análisis de cómo se abordó este específico problema en la fortificación de los puertos de Jávea y de Moraira en la segunda mitad del s. XVI permite la confrontación de las diversas soluciones tipológicas empleadas en las torres que se propusieron a tal fin.

\section{De las torres-atalaya a las torres-fortaleza. Los proyectos de Giovanni Battista Antonelli para los puertos de Jávea y de Moraira (1553- 1563)}

En 1563 el ingeniero militar italiano al servicio de la Monarquía Hispánica Juan Bautista Antonelli en su discurso sobre la fortificación y defensa del Reino de Valencia -escrito después de los dos viajes de reconocimiento realizados en 1561 y 1562- recogía la primera relación de las torres existentes en la costa valenciana levantadas pocos años antes por el duque de Maqueda. J.B. Antonelli valoraba relativamente el funcionamiento de estas torres en la medida que también suponían un significativo avance sobre el anterior sistema autónomo de vigilancia medieval, pero consideraba que, además de insuficientes, eran inadecuadas para las nuevas exigencias de la guerra moderna: «porque estas torres no sirven si no para señalar, lo cual no basta enteramente para desechar los enemigos, pues se ponen en las calas que ay fuera de tiro de arcabuzes...haciendo aguadas y leñas como quieran, e muchas partes desta costa, sin que sean parte las torres para impedírselo, ni aun para defenderse ellos mismos» ${ }^{1}$. Era pues necesario superar las limitaciones de un sistema preventivo de vigilancia con nuevas torres cuya acción ofensiva posibilitara el rechazo de los navíos enemigos. Para J.B. Antonelli la finalidad era conseguir un control eficaz de todos aquellos puntos débiles con una fortificación artillada que evitara los posibles desembarcos: «en las partes en donde han desembarcado hasta agora, y que puedan desembarcar, habrá torres o lugares, que los tiros de la una torre o lugar alcançaran por la mayor parte de esta costa el tiro de la otra, de manera que el enenmigo no osará ponerse entre los tiros» ${ }^{2}$.

Así, las propuestas de J.B. Antonelli pasaban por la reconversión de las esbeltas y débiles torres 
vigía en efectivos puntos de fuerza ofensiva mediante una adecuación constructiva -con refuerzos y guirnaldas- que permitiera acomodar en ellas el uso de piezas de artillería: «en donde ay torres hacerles sus defensas y remediarlas de manera que puedan en cada una caber uno o dos morteretes o pedreros, la qual suerte de pieças ha parescido la mejor para las dichas torres. Estas torres fortificadas $y$ artilladas de.sta manera y con la de los lugares no dexarán recoger los enemigos en ninguna cala deste reyno... los navios christianos se podrán recoger siempre debaxo de cualquier torre destas y ser deffendidos de artillería $»^{3}$.

En la torre que existía sobre el cabo de Moraira, proponía construir una guirnalda y dotarla de un mortero cuyo tiro parabólico podría resultar de utilidad para cubrir la ensenada que quedaba oculta a la visual de la torre.

J. B. Antonelli, en su informe de 1563, fue el primero en proponer la fortificación de los puertos de Jávea y Moraira que a su juicio eran los de mayor consideración después de los principales del reino al tratarse de puertos naturales muy capaces y a menudo utilizados para abrigo y desembarcos enemigos. De este modo entre las torres a construir de nueva planta propuso la ejecución de torres en estos dos puertos, situados bajo el resguardo de los promontorios del cabo de San Antonio y del cabo de Moraira, ubicándose la primera en el llamado «rincón de Sancto Antón» en Jávea y la otra en el «toçalete de Miguelete en lo más alto junto a la mar» en el puerto de Moraira.

Las nuevas torres proyectadas en estos lugares contarían ya con una concepción adaptada a su finalidad y por su importancia deberían ser especialmente fuertes y pensadas básicamente en función de la artillería que deberían albergar, ya que la función de vigilancia quedaba asistida por las torres ubicadas en altura sobre dichos cabos.

El discurso de J. B. Antonelli constata la pretensión que ya tenían los habitantes de Jávea para construir una torre en el puerto: «los de Xabea ya tienen hecho un castillo y agora tratan de hazer otro para assegurar el rincón de Sanct Antón» ${ }^{4}$. Antonelli proyectó esta torre en el invierno de 1562 durante el segundo viaje que realizó a las costas valencianas proporcionando su traza al consejo de la villa en su visita a Jávea: «El rincón de Sanct Antón donde están unas paredes viejas se hará un castillo conforme a la traça que se ha dexado a los de Xabea para guardar aquel puerto guardado de tramontana y levante por quitar aquella comodidad a los enemigos de poder con ella dañar a las dos villas de Denia y Xabea» ${ }^{5}$.

En marzo de 1562 ya se concedía a los jurados de la villa la cesión de los derechos de puerto para la financiación de las obras otorgándose las amplias facultades para edificar (Espinós, Polo, 1985). Sin embargo la suspensión del proyecto de J.B. Antonelli para la defensa del Reino solicitado por las instituciones valencianas, que consideraron inasumible su coste, y el diferente parecer que para su fortificación tuvo después el virrey y experto militar Vespasiano Gonzaga, retrasaron la financiación de la obra hasta después de $1579^{6}$.

La traza prevista para la torre del puerto de Moraira era la misma que la facilitada a Jávea puesto que los rasgos geográficos de ambos puertos eran muy similares: «En el puerto de Morayra se hará una torre conforme a la traça que se dio a los de Xabea por el rincón de San Antón y se proveerá de la guarda y artillería necesaria $»^{7}$. Sin embargo las diferencias más importantes radicaban en las condiciones de tierra, esto es, en la más importante población de la villa de Jávea y su mayor proximidad al puerto respecto de la menor población y relativa lejanía del mar de la villa de Teulada. Factores que a la postre serían determinantes para que la torre del puerto de Moraira no llegara a construirse.

A pesar de su desaparición en el siglo XIX conocemos su traza a partir las vista topográfica del cabo de San Antonio que por encargo real realizó el paisajista Mariano Sánchez en 1787 y de la descripción que de ella realizó Juan de Acuña en 1585: «La torre de la Mesquida está de la villa de Jabea un quarto de legua, está edificada junto a la mar al pie de la subida de la sierra del cabo de Sant Antón, es nueva y muy buena, y se hizo (...) con buen escarpe hasta la 
mitad, y tiene una guirnalda encima por donde se guarda el pie de.lla, la qual está sobre canes de piedra en que carga el parapeto, súbese a la puerta por escala de mano a la mitad, tiene una ventana pequeña para luz» ${ }^{8}$. Se trataba de una torre de forma hexagonal y de mayor tamaño que las existentes para ser guardada ordinariamente por cuatro hombres durante el día y acudir desde Jávea seis guardas durante la noche. En realidad se trataba de una pequeña fortaleza más que de una torre, pues el mismo Antonelli se refiere a ella también como «castillo» o «castillejo» y preveía que estuviese fuertemente artillada con un pedrero y dos morteretes. Además debía de dotarse con 6 mosquetes y 12 arcabuces. La traza se correspondería con la de las torres hexagonales que había propuesto para el Reino de Murcia y que se ejecutaron en 1578 Sin duda, con esta dotación humana y de artillería, la traza de J.B. Antonelli para el puerto de Jávea contemplaba la misma tipología hexagonal y dimensiones que las torres que realizaría después en Murcia. Estas constaban de dos cámaras superpuestas sobre una base escarpada en terraplén y se remataban por una guirnalda volada sobre ménsulas de piedra (Cámara, 1991).

Sin embargo de la descripción de Acuña y de las proporciones representas en la pintura descriptiva y realista de Mariano Sánchez se deduce que la edificación de la torre del puerto de Jávea, veinte años después, se ejecutó finalmente con variaciones sobre la traza inicial de J.B. Antonelli que redujeron las dimensiones y dejaron una sola cámara abovedada en correspondencia también con la menor dotación de efectivos -medio falconete y tres guardas- finalmente realizada. No obstante estas variaciones mantuvieron, aunque reducida, su forma originaria, y creemos que se deben al ingeniero real Cristóbal Antonelli sobrino y discípulo de Juan Bautista que, como después se verá, se hizo cargo en los primeros años de 1580 de estas obras en el litoral valenciano. Así pues, J.B. Antonelli en el temprano proyecto para las torres hexagonales de los puertos de Jávea y Moraira adelantaba soluciones -efectuadas después en la costa de Murcia-que no se implantarían de forma sistemática en la concepción de las torres marítimas hasta finales del siglo XVI.

\section{Las propuestas tipológicas de $\mathbf{1 5 9 6}$ para la torre del puerto de Moraira}

El proyecto de J.B. Antonelli de edificación de una torre que defendiera el puerto de Moraira permanecería olvidado - del mismo modo que la mayor parte de las actuaciones que propuso para la defensa del reino valenciano- hasta que en 1585 el capitán Juan de Acuña, en la visita y relación que por mandamiento real hizo sobre la costa valenciana, señaló nuevamente esta necesidad (Banyuls, Boira, Lluesma, 1996). Acuña proponía edificar para este puerto una torre que debería ser especialmente fuerte, capaz para tres piezas grandes de artillería y cuyo coste de construcción podría alcanzar los tres mil trescientos ducados. Esta torre, sin embargo, no se materializaría en un proyecto concreto hasta 1596, cuando se habían reactivado nuevamente las obras para poner a punto la fortificación de la de la parte de poniente de la costa valenciana impulsadas en el virreinato de Francisco de Moncada, marqués de Aytona y conde de Osona (1580-1595) y por su sucesor el marqués de Denia y conde de Lerma -después duqueFrancisco de Sandoval y Rojas, nombrado virrey en junio de 1595.

Desde 1583, y probablemente a recomendación de Juan Bautista Antonelli, también había sido designado para ocuparse de las obras de fortificación y del real patrimonio en el Reino de Valencia el ingeniero al servicio real Cristóbal Antonelli (Llaguno, 1829). En la costa valenciana el ingeniero se encargaría de algunos de los antiguos proyectos de su tío y maestro como la torre del puerto de Jávea iniciada en esas fechas. En 1585 se ocupaba de proyectaba las nuevas murallas para la villa de Benidorm ${ }^{9}$. Entre 1587 y 1596 intervenía en las obras de la presa del pantano de Alicante en Tibi, del que también se había ocupado anteriormente su tío (Alberola, 1984), y había reconocido la costa de Altea para reedificar de nueva planta esa villa (Gutierrez del Caño, 1980). A su vez, entre 1590 y 1595 se ocupó de los reparos y proyectos de la torres de la costa de poniente como la torre de la 
cala Horadada en Orihuela, la torre de la Santa Faz en Alicante y de la reedificación, con nueva traza, de la derruida torre sobre el cabo de Moraira (Banyuls, Boira, Lluesma, 1996).
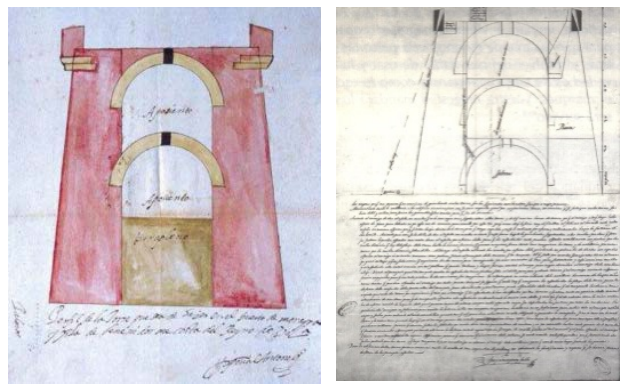

Fig. 2- Proyectos de Cristobal Antonelli y fray Ximén Pérez Fachs para la torre del puerto de Moraira, ca. 1596 (España, M.E.C.y D. Archivo de la Corona de Aragón, MP-23/1 y MP-23/3)

Poco después del nombramiento del marqués de Denia como virrey de Valencia este visitó, en noviembre de 1595, las torres de la costa de poniente constatando la urgente necesidad de realizar aquellas propuestas, señaladas desde muchos años antes, para edificar las torres para el puerto de Moraira -J.B.Antonelli en 1563 y Acuña en 1585- y para la isla de Benidorm ${ }^{10}$ -Vespasiano Gonzaga en 1575-. Poco después de la visita del virrey Cristóbal Antonelli tenía realizada la traza para la construcción de estas dos torres. Sin embargo, en marzo de 1596, el marqués de Denia había hecho confrontar, de forma interesada, el proyecto de Cristóbal Antonelli con la opinión y traza que paralelamente había solicitado el marqués para dichas torres al comendador de la orden militar de San Juan fray Ximén Pérez Fachs «que es matemático y muy pláctico en las fortificaciones a lo moderno» ${ }^{11}$. Una vez más, los distintos pareceres sobre el diseño de la fortaleza fueron objeto de controversias y recelos profesionales entre expertos en la nueva ciencia de fortificación, pero también es posible intuir una subyacente pugna de poder del marqués de Denia que fue perfectamente entendida por Felipe II a la luz de la categórica y concisa resolución a favor del ingeniero real. Ambos proyectos trataban de torres muy capaces y fuertes, en realidad reducidas fortalezas, pensadas en función de la artillería, tanto para ser menos vulnerables al impacto de baterías y de las minas de fuego como para soportar sobre ellas el juego de las piezas de artillería. Este modelo de torre tenía su antecedente inmediato en la tipología de las torres que en 1577 había hecho edificar Vespasiano Gonzaga en el litoral valenciano: de planta circular con un diámetro inferior similar a su altura con toda su elevación en talud $\mathrm{y}$ no sólo en su base, como anteriormente habían sido proyectadas por J.B. Antonelli, con la finalidad de otorgarle una mayor estabilidad y fortaleza

El proyecto de Cristóbal Antonelli para el puerto de Moraira seguía prácticamente el mismo tipo que ya propuso unos años antes, en 1591, para la torre de la cala Horadada en Orihuela cuyo coste era muy similar al que se estimó para la torre de Moraira en 1585 . Su traza era redonda, de 53 palmos de diámetro en la base y 55 palmos de altura hasta la plataforma superior. El hueco interior era de 21 palmos y terraplenado en el tercio inferior de su altura total. Disponía de dos salas abovedadas superpuestas, la primera -donde se guardaría la munición- tendría 20 palmos de altura y la superior -que alojaría la chimenea- sería de 15, incluyendo en esta el grosor de las bóvedas que preveía de 2,5 palmos. Como en Orihuela, las cámaras estarían comunicadas, además de con una escalera de piedra labrada, también por un paso abierto en el centro de las bóvedas con la finalidad de: «que per allí puguen pendre llum les dites dos peses per dins la mateixa torre per no obrir espillera ni finestra...per major fortificació de aquella» ${ }^{12}$. Los muros tendrían un espesor de 16 palmos en la base, disminuyendo en altura de cada diez palmos uno para conformar el talud. De esta manera resultaba una plataforma superior de un diámetro de 41 palmos. La torre se coronada con una guirnalda formada por un parapeto de 5 palmos de espesor y 5 de altura que descansaba sobre dos órdenes de ménsulas de piedra labrada que volaban 3 palmos por fuera del cuerpo de la torre. El parapeto se anclaba, empotrándose los 2 palmos restantes, a la fábrica de la torre dejando de este modo una plataforma libre para el uso de la artillería de 37 palmos de diámetro. En el espesor del parapeto se abrirían tres o cuatro 
troneras orientadas convenientemente para actuar las tres piezas de artillería que preveía.

La torre que proponía el comendador Fachs presentaba una traza similar en proporciones y coste a la proyectada por Antonelli: troncocónica con un diámetro en la base algo más ancha -60 palmos de diámetro- y de 54 palmos de altura hasta la plataforma. Superiormente estaba rematada por un parapeto que dejaba una plataforma libre para el juego de la artillería de 40 palmos de diámetro. Disponía para su uso también de dos cámaras superpuestas cubiertas con bóveda de ladrillo, pero comunicadas ahora por escaleras de mano a través de los huecos de iluminación abiertos en la clave de las bóvedas para evitar debilitar los muros con escaleras empotradas en ellos. De la misma forma que en la traza de C. Antonelli, la del comendador Fachs prescindía de toda apertura en el cuerpo de la torre a excepción de la inevitable puerta de acceso situada a la altura del primer piso. La inutilidad i rechazo del uso de aspilleras se explicaba en el parecer del comendador: «en el tiempo que el de adentro toma la mira contra los de afuera rescibe las rosziada de la qual parece imposible poderse escapar nadie y por esta razón son desechadas las saeteras por todos los modernos» ${ }^{13}$.

Aún así, la traza de fray Ximén Pérez Fachs tenía diferencias cualitativas respecto de la propuesta de Cristóbal Antonelli ya que concebía una tipología de torre de mayor anchura -que superaba ya su altura total-y más escarpada, perdiendo en altura uno de cada siete palmos. El núcleo terraplenado en la base se sustituía por un aljibe, dotando a la torre de mayor autonomía, a la vez que -según argumentaba el comendador- favorecía su protección frente a la tentativa de minar la torre por la base. Además preveía reforzar la bóveda superior con una rosca de mayor grosor -4 palmos- que arrancaba más baja con el fin de fortalecer la plataforma superior y compensar el menor grosor que tenían los muros en este nivel debido a la mayor inclinación del talud. No obstante, lo más destacable de su controvertido proyecto era la crítica que realizaría al uso de las guirnaldas: «Atormentando tanto la artillería a los edificios...no conviene que se fabrique en las torres fábrica débil y costosa como serían las guirnaldas sino macisa para que sea de durada» ${ }^{14}$. Además esta valoración negativa de las guirnaldas también la fundaba en qué el ataque que con mayor probabilidad podían sufrir estas torres era el asalto con escaleras de mano, donde el enemigo trataría de evitar la ofensiva de las troneras ubicadas en la guirnalda y por ello «antes dará el asalto por afuera de ella». Según Fachs, el rechazo a este tipo de asalto se vería también dificultado por los gruesos muros de los parapetos, propuestos por C. Antonelli, que impedirían a los defensores de la torre poder acceder a las escaleras apoyadas por fuera de la guirnalda.

Los nuevos sistemas de fortificación se encontraban en continua evolución lo que se manifestaba en las continuas críticas realizadas sobre las obras precedentes. Así, fray Ximén Pérez Fachs cuestionaba la eficacia de las modernas guirnaldas que tanto habían defendido expertos como J. B. Antonelli o Vespasiano Gonzaga para las nuevas torres marítimas construidas pocos años antes. Como alternativa a la guirnalda el comendador proponía en su traza la solución de un parapeto más delgado que volaba por fuera del cuerpo de la torre - sin ménsulas- sólo lo necesario para poder abrir pequeñas aspilleras empotradas en el mismo espesor que impidieran picar en su base. Este corto voladizo permitiría una unión más sólida a la fábrica de la torre sin necesidad de construir parapetos tan gruesos como los que proyectaba C. Antonelli. De esta manera su espesor seria sólo el necesario para poder resistir el tiro más probable de mosquetes.

La altura de los parapetos también era cuestionada por el comendador Fachs. Opinaba que los parapetos altos protegían a los que hacían guardia encima de la torre, pero impedían la eficacia en la orientación de las piezas de artillería, por lo que se tenían que abrir numerosas cañoneras en todas las direcciones con la consiguiente creación de múltiples puntos débiles por donde se sufría habitualmente la agresión enemiga: "Confiados de las partes altas del parapeto serán descubiertos y en el mesmo punto offendidos de los tiradores que de mosquete $^{15}$ les darán una roziada qual cada día 
vemos». También opinaba que los parapetos tenían que ser menos altos para que la artillería «corra a barba» de su remate, aumentando de esta forma su operatividad aunque con ello la torre quedase más expuesta desde tierra, sin embargo, a su parecer, ello era accesorio a su primordial finalidad: «el que guardara estas torres no está obligado a guardar la campaña si no la mar con la artillería» ${ }^{16}$. Cristóbal Antonelli proponía en su modelo, rebatiendo esta cuestión, prever el uso de tablones móviles mientras las troneras del parapeto no estuvieran siendo utilizadas: «se dexaran tres o quatro troneras arriba parar tirar la artillería con unos encaxes para poner unos tablones de quatro ho cinco dedos de grueso para quitar y poner (...) para que no sean vistos desde fuera estando cargando la artillería no sea offendida $»^{17}$.

Por último, el comendador Fachs también aconsejaba trasladar a la plataforma superior el depósito de pólvora «porque sucediendo desastre padezcan poco las torres», recomendación, que junto a la de ubicar el aljibe en su base, ya había sido realizada por Vespasiano Gonzaga en 1570 para las torres que proponía edificar en Murcia (Cámara, 1991). Contrariamente a la opinión del comendador C. Antonelli defendía el uso de las guirnaldas y criticaba la propuesta de Fachs, amparándose en la inoperancia de aquellas fortificaciones que, como la fortaleza de Altea, no disponían de un parapeto con suficiente grosor y altura para proteger arriba a los soldados y artilleros: «le $a$ parecido en lugar de guirnalda haçer un cordon en dichas torres que salga afuera no más de un palmo con unos agujeros muy pequeños para solo ver a los que queran minar o picar la torre y no para ofenderles por allí, y con un parapeto muy delgado y baxo que no abrá quien esté a la defensa y esto se ha visto por esperiençia en la fortaleça de Altea ques en la costa deste Reyno que por estar el parapeto bajo los moros escopeteaban pordefuera y no avía quien se pudiesse asomar para poder tirar el artillería y assi se llevaron un navío que avía junto a la fortaleça, lo mesmo ycieron en la forteça qu ay en los Alfacs de Tortosa estando yo ally» ${ }^{18}$.

También a su favor apelaba a la autoridad de los prestigiosos expertos que se habían ocupado anteriormente de las torres marítimas en los reinos de Murcia y Valencia, como lo fue su tío, J. B. Antonelli -introductor de este elemento arquitectónico en las torres del litoral valenciano- y reportaba el ejemplo de las nuevas torres edificadas en la costa valenciana desde el tiempo del virrey Vespasiano Gonzaga «que son seys ho siete que paresçe que no ay otras mejores».

Ante la discrepancia de opiniones de los dos expertos, las trazas de las torres, las modelos de madera y los pareceres escritos de ambos expertos, fueron remitidas por el marqués de Denia al Rey, para que se decidiera por uno de las dos propuestas a la vez que solicitaba una respuesta urgente dada la necesidad de acometer rápidamente las obras.
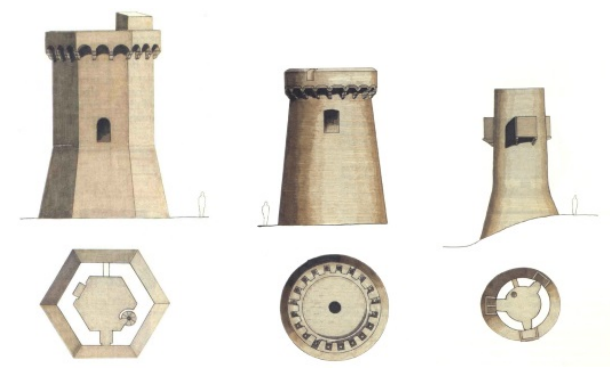

Fig. 3- Tipologías de torres marítimas: puerto de Jávea (1562/1584), cabo de Moraira (1595) y cabo de San Martín en Jávea (1554) (Antoni Banyuls, 1995)

En septiembre de 1596, en el seno del Consejo de Aragón, se resolvió, sin razonamiento escrito, ni mención alguna al proyecto de Fachs, ordenando la ejecución de la traza del ingeniero real Cristóbal Antonelli. Sin embargo y pese a la importancia y urgencia dada para la edificación de esta torre, junto a la de la isla de Benidorm -ambas de la misma traza- nunca se llegaron a construir. Las dificultades financieras de $1597 \mathrm{y}$, sobretodo, la sucesión en la Corona a finales de 1598 -que reportaría al marqués de Denia un ascenso político sin límites- seria determinante en la paralización definitiva del proyecto. Desde 1599 los intereses financieros para el ya duque de Lerma y «valido» de Felipe III eran otros.

De este modo la nueva torre artillada reedificada sobre el cabo de Moraira tuvo que desempeñar, 
también la función de control del puerto. Su reconstrucción -muy probablemente efectuada entre 1595 y 1596- supuso una total renovación morfológica de la vieja y semiderruida torre y respondía a la moderna concepción de torres artilladas: troncocónica en toda su altura y coronada por una guirnalda volada. Pero por su función preponderante de avistamiento en altura se trataba de una torre de las consideradas pequeñas con una única sala abovedada. Juan de Acuña en 1585 calculaba su coste en 800 ducados, es decir, la cuarta parte del presupuesto que se estimaba para la torre grande del puerto. $\mathrm{La}$ torre se reconstruyó, para una mayor fortificación e inaccesibilidad, terraplenado los dos tercios de su altura y probablemente como envoltura de los restos existentes de la arruinada torre edificada en tiempo del duque de Maqueda.

\section{Notas}

(1) Archivo General de Simancas. E., 329-I, f.2r.

(2) Ibídem, f. 3r.

(3) Ibídem, f. 2v-3r

(4) Ibídem, f. 7v
(5) Ibídem, f. 13v

(6) Las obras se no se pudieron iniciar hasta finales de 1582 concluyéndose en 1584 (Arciniega, 2001, II: 235).

(7) Ibídem, f. 7r

(8) Archivo de la Corona de Aragón. Consejo de Aragón, leg. 0761, n 103, f. 12r.

(9) Ibídem, f. 9v

(10) Archivo de la Corona de Aragón Consejo de Aragón, leg. 0560, n³7, f. 1r.

(11) Ibídem.

(12) Archivo Municipal de Orihuela. Torres de la Costa, 1039.

(13) Archivo de la Corona de Aragón Consejo de Aragón, leg. 0560, n³7, f. 3r.

(14) Ibídem.

(15) Ibídem.

(16) Ibídem.

(17) Archivo de la Corona de Aragón Consejo de Aragón, leg. 0560, n³7, f. 2v.

(18) Ibídem, f. 3r.

\section{Referencias}

Alberola A. (1984). El pantano de Tibi y el sistema de riegos de la huerta. Institut de Cultura Juan GilAlbert Ed. Alacant. p. 42.

Arciniega L. (2001). El Monasterio de San Miguel de los Reyes. Biblioteca Valenciana Ed. Valencia. Vol. II, p. 235.

Banyuls A., Boira J.V., Lluesma A. (1996). Arquitectura i control del territori: la defensa del litoral de la marina Alta al segle XVI. Institut de Cultura Juan Gil-Albert Ed. Alacant. pp. 34-37.

Boira J.V. (2007). Las torres del litoral valenciano. Conselleria d'Infraestructures i Transport Ed. Valencia. pp. 27-41.

Cámara A. (1991). "Las torres del litoral en el reinado de Felipe II: una arquitectura para la defensa del territorio ( y II)” en Espacio, Tiempo y Forma, Serie VII, Hª del Arte, T.4 Ed. Madrid. p. 61-62.

Espinós A., Polo F. (1985). Xàbia. Anotaciones históricas de una villa mediterránea. Ajuntament de Dénia Ed. Alacant. p.181.

Gutiérrez del Caño M. (1920). Monografía histórica de la villa de Altea. La Voz Valenciana Ed. Valencia. p. 211.

Llaguno E. (1829). Noticias de los arquitectos y arquitectura de España desde su restauración. Imprenta Real Ed. Madrid. Vol. III, p.45. 